\section{Accurate profile extraction and mapping by intelligent processing of ultrasonic range data}

\section{B. Barshan}

A novel technique is described that employs intelligent processing of multiple ultrasonic range measurements for accurate surface profile extraction and mapping. It aims to overcome the intrinsic angular uncertainty of ultrasonic sensors, as well as eliminating noise and crosstalk related misreadings. Experimental verification is provided where the ultrasonic range data is acquired through wall following. The technique is evaluated based on three complementary error criteria. Compared to the most recently reported arc-transversal median algorithm, the proposed technique provides about $50 \%$ smaller mean absolute error, $28-50 \%$ higher correct detection of pixels as full and empty, and 10-15 times smaller CPU time, which is less than a tenth of a second. The method is also superior in eliminating artefacts.

Introduction: The utilisation of simple sensors together with appropriate processing of acquired data can go a long way in aiding intelligent systems to become aware of their environment. The potential of simple and inexpensive sensors should be fully exploited before more expensive alternatives with higher resolution and resource requirements are considered for a given task. Ultrasonic sensors have been widely employed owing to their accurate range measurements, robustness, low cost, and simple hardware interface, e.g. their use in phased arrays for non-destructive testing and evaluation has increased considerably in recent years [1]. We explore the limits of simple and low-cost ultrasonic sensors in profile extraction and mapping through intelligent processing of raw ultrasonic data. Ultrasonic sensors, when coupled with intelligent processing, are a useful alternative to more complex laser and camera systems. Furthermore, it may not be possible to use the latter in some environments owing to surface characteristics or insufficient ambient light. Despite their advantages, the frequency range at which airborne ultrasonic transducers operate is associated with large beamwidth that results in low angular resolution and uncertainty in the location of the echo-producing feature. Thus, having an intrinsic uncertainty of the actual angular direction of the range measurement and being prone to various phenomena such as multiple and higher-order reflections and crosstalk between transducers, a considerable amount of modelling, processing, and interpretation of ultrasonic data is necessary.

Representing angular uncertainty by ultrasonic arc maps: Simple ultrasonic range sensors are considered that measure the time-of-flight $t_{o}$, which is the round-trip travel time of the transmitted pulse between the transducer and the point of reflection. Given the speed of transmission $c$, the range $r$ can be calculated from $r=c t_{o} / 2$. Although such devices return accurate range data, typically they cannot provide direct information on the angular position of the reflection point on the surface. Most commonly, the large beamwidth of the transducer is accepted as a device limitation that determines the angular resolving power of the system, and the reflection point is assumed to be along the line-of-sight (LOS). According to this naive approach, a simple mark is placed along the LOS of the transducer at the measured range, resulting in inaccurate maps with large angular errors. Alternatively, the angular uncertainty in the range measurements can be represented by ultrasonic arc maps (UAMs) that preserve more information (see Figs. $2 d-f$ for some examples). This is done by drawing arcs spanning the beamwidth of the transducer at the measured range, indicating that the echo-producing feature can lie anywhere on the arc [2]. Thus, when the same transducer transmits and receives, all that is known is that the reflection point lies on a circular arc of radius $r$ (Fig. 1a). More generally, when one transducer transmits and another receives, it is known that the reflection point lies on the arc of an ellipse whose focal points are the transmitting and receiving elements (Fig. 1b). The arcs are tangent to the reflecting surface at the actual point(s) of reflection. Arc segments near the actual reflection points tend to reinforce each other. Arc segments not actually corresponding to any reflections and simply representing the angular uncertainty of the transducers, remain more sparse and isolated. Arcs generated by spurious readings, crosstalk, and higher-order reflections also remain sparse and lack reinforcement. The proposed method is capable of effectively suppressing these effects, and has the intrinsic ability to process echoes returning from surface features further away than the nearest (i.e. multiple reflections) informatively. By combining the information inherent in a large number of such arcs, angular resolution far exceeding the beamwidth is obtained.

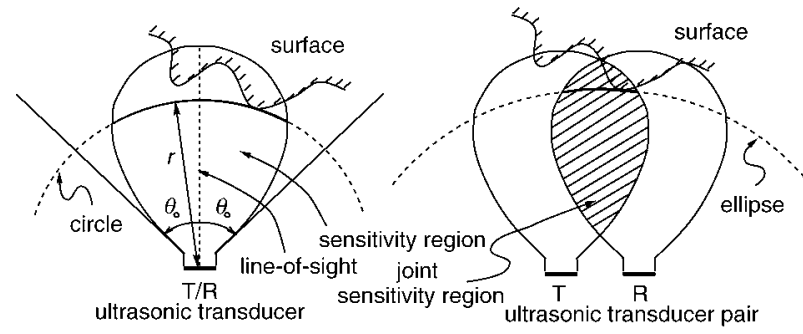

a

$b$

Fig. 1 Ultrasonic transmission and reception

$a$ For same transducer transmitting and receiving, reflection point known to be on circular arc shown

$b$ Elliptical arc if wave transmitted and received by different transducers
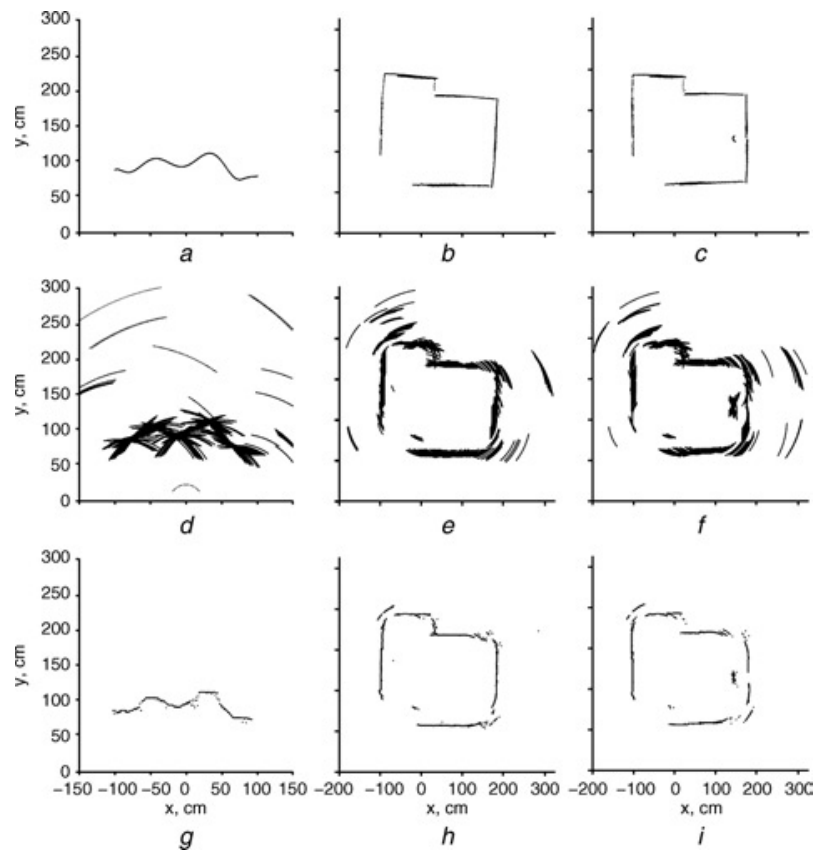

Fig. 2 Laser data acquired from three environments; corresponding UAMs; resulting maps

$a-c$ Laser data acquired from three environments

$d-f$ Corresponding UAMs

$g-i$ Resulting maps

Processing the UAM: The proposed technique introduces a sense of direction in data acquisition and processing. Such directional awareness can be cost-effective and beneficial [3]. The direction(s)-ofinterest (DOI) are those directions along which the UAM is processed, ideally corresponding to the surface normals of the environment being explored. However, in practice, since prior information on the environment is usually unavailable, the DOI needs to be estimated while sensory data are being collected and processed online. It may also be possible to determine the DOI by post-processing, based on the distribution of the already acquired data.

Using the UAM, the number of arcs crossing each pixel is counted and stored, and a suitable threshold value chosen. The array of pixels along each DOI is inspected and the pixel with the maximum count that also exceeds the threshold is kept, while remaining pixels along the DOI are zeroed out. If there exist more than one maxima, their median is taken (if the number of maxima is even, one of the two middle maxima is randomly selected). This way, most of the artefacts of the UAM can be removed.

Experimental results: The experiments were performed using the Polaroid 6500 series ultrasonic transducers [4] available on the Nomad 200 mobile robot. This transducer has a resonance frequency 
of $f_{o}=49.4 \mathrm{kHz}$ and aperture radius $a=2.0 \mathrm{~cm}$, corresponding to a half-beamwidth angle of $\theta_{o}=\sin ^{-1}\left[0.61 c / a f_{o}\right]=12.2^{\circ}$. Results from three sample experiments are provided where ultrasonic and laser data are collected simultaneously. Both systems are rigidly fixed to the turret of the mobile robot so that the correspondence between them is never altered. The laser system is much more expensive and complex, requiring higher power and sufficient ambient light for operation. Furthermore, this mode of sensing does not work in all environments, such as those with dark-coloured upholstery and glass. Since it reveals a very accurate surface profile, the profile detected by the laser is taken as an absolute reference in the evaluation of the proposed technique.

First, curved surfaces were constructed in our laboratory with varying curvature and dimensions, using thin cardboard of dimensions $1.05 \times 3.65 \mathrm{~m}$. In these experiments, only the front five ultrasonic sensors of the robot were fired. The laser data obtained from one of the cardboard surfaces constructed are presented in Fig. $2 a$. As expected, the laser data provide a very accurate surface profile. In this experiment, the mobile robot simply translated along a straight path from $(-75,0) \mathrm{cm}$ to $(75,0) \mathrm{cm}$ alongside the surface at an average distance of $1 \mathrm{~m}$ and collected data by firing the ultrasonic transducers at every $2.5 \mathrm{~cm}$. The turret is oriented such that both the laser and the front five ultrasonic sensors are directed towards the surface and it does not rotate throughout the translational movement. Therefore, the DOI for this example is taken to be the positive $y$ direction, perpendicular to the path of the robot. The environment is divided into $300 \times 300$ square elements or pixels with size $1 \mathrm{~cm}$. In the resulting UAM shown in Fig. $2 d$, there are some arcs which are not tangent to the actual surface at any point (e.g. the isolated arcs in the upper part of Fig. 2d). These correspond to spurious data owing to higher-order reflections, readings from other objects in the environment, or totally erroneous readings. The result of processing the curved surface data with a threshold value of 4 is presented in Fig. $2 g$. The highly populated UAM given in Fig. $2 d$ is successfully cleaned and the branches and dark regions have been removed to give a sufficiently accurate surface profile. Only a few minor point artefacts remain.

We also present experimental results obtained by following the walls of the indoor environment in Fig. $2 b$. The environment comprises smooth wooden (top and left) and painted (right) walls, and a window shade with vertical slats of $15 \mathrm{~cm}$ width (bottom). Some of the corners of the room are not perfect. The resulting UAM is given in Fig. $2 e$. Again, the UAM includes many artefacts, especially exterior to the surrounded region where the robot can move. In Fig. $2 c$, a cylindrical object of radius $15 \mathrm{~cm}$ has been added to the environment at a distance of $30 \mathrm{~cm}$ from the centre of the right wall. The results of these two experiments are shown in Figs. $2 h$ and $i$. In these two experiments, we used a simple rule-based wall-following scheme for motion planning and took the DOI as the direction of the currently followed wall, i.e. the DOI is perpendicular to the path of the robot and is in the direction of the nearest wall from which a TOF reading is acquired.

The performance evaluation is based on three complementary error criteria which are more meaningful when considered together. These are the mean absolute error (MAE) between the acquired map and the laser map, the correct detection rate (CDR) of borderline pixels as full and empty, and the computational cost in terms of CPU time. The processing technique has been implemented in the $\mathrm{C}$ language and run on an Intel Pentium 4 PC with $3.00 \mathrm{GHz}$ Hypertreading processor and $1 \mathrm{~GB}$ memory. Internal $\mathrm{C}$ commands are used for time keeping. The results of the three sample experiments are presented in Table 1. A comparison is provided (based on the same data) with the most recently reported arctransversal median (ATM) algorithm for processing UAMs [2]. The technique described here provides about $50 \%$ smaller MAE, $28-50 \%$ higher CDR, and 10-15 times smaller CPU time compared to the ATM algorithm. The CPU times involved are less than a tenth of a second, indicating that the method is viable for real-time applications.

Table 1: Results of three sample experiments

\begin{tabular}{|l|c|c|c|c|}
\hline & No. of arcs processed & MAE (pixels) & CDR (\%) & CPU time (s) \\
\hline Experiment 1 & 287 & $\mathbf{2 . 2 3}(4.33)$ & $\mathbf{9 6 . 0}(74.8)$ & $\mathbf{0 . 0 3 1}(0.310)$ \\
\hline Experiment 2 & 697 & $\mathbf{2 . 3 6}(4.47)$ & $\mathbf{9 3 . 8}(62.4)$ & $\mathbf{0 . 0 8 5}(1.126)$ \\
\hline Experiment 3 & 659 & $\mathbf{2 . 3 7}(4.78)$ & $\mathbf{8 8 . 3}(60.6)$ & $\mathbf{0 . 0 7 8}(1.054)$ \\
\hline
\end{tabular}

Results based on three complementary error criteria in bold. ATM results given in parentheses

Conclusions: A new technique is described for processing UAMs which is robust and flexible in that it can be applied to ultrasonic data acquired with many different transducer configurations. It has the inherent ability to eliminate undesired range readings arising from higher-order reflections, crosstalk, and noise, as well as processing multiple echoes informatively. The new technique is much superior to the existing ATM technique in terms of MAE, CDR, and CPU time. The method can be readily generalised to three-dimensional environments with the arcs replaced by spherical or elliptical caps and the processing rule extended to three dimensions. In certain problems, it may be preferable to reformulate the method in polar or spherical co-ordinates.

Acknowledgment: This work is supported by the Scientific and Technological Research Council of Turkey (TÜUBITAK) under grant number EEEAG-105E065.

(C) The Institution of Engineering and Technology 2007

21 June 2007

Electronics Letters online no: 20071828

doi: 10.1049/el:20071828

B. Barshan (Department of Electrical Engineering, Bilkent University, Bilkent, Ankara 06800, Turkey)

E-mail: billur@ee.bilkent.edu.tr

\section{References}

1 Drinkwater, B.W., and Wilcox, P.D.: 'Ultrasonic arrays for nondestructive evaluation: a review', NDT\&E Int., 2006, 39, (7), pp. 525-541

2 Choset, H., Nagatani, K., and Lazar, N.A.: 'The arc-transversal median algorithm: a geometric approach to increasing ultrasonic sensor azimuth accuracy', IEEE Trans. Robot. Autom., 2003, 19, (3), pp. 513-522

3 Barshan, B.: 'Directional processing of ultrasonic arc maps and its comparison with existing techniques', Int. J. Robot. Res., 2007, 26, (8), pp. $797-820$

4 Polaroid Corporation: Ultrasonic Components Group, Polaroid Manual, 119 Windsor St., Cambridge, MA, 1997 